\title{
Erratum to: The all-source Green's function (ASGF) and its applications to storm surge modeling, part II: from the ASGF convolution to forcing data compression and a regression model
}

\author{
Zhigang Xu ${ }^{1}$
}

Published online: 21 December 2015

(C) Springer-Verlag Berlin Heidelberg 2015

Erratum to: Ocean Dynamics (2015) 65:1761-1778

DOI 10.1007/s10236-015-0894-y

The original version of this article unfortunately contained a mistake. The first three lines on page 1778 should be corrected as follows: where $\mathbf{U}_{\mathbf{1}}$ and $\mathbf{U}_{\mathbf{2}}$ are the first and second columns of $\mathbf{U}, \mathbf{s}=\left[\begin{array}{ll}s_{1} & s_{2}\end{array}\right]^{T}$, and $\psi_{1}$ and $\psi_{2}$ are defined by:

$$
\boldsymbol{\psi}_{\mathbf{1}}=\left[\begin{array}{l}
\psi_{1}^{(0)} \\
\psi_{1}^{(1)}
\end{array}\right], \quad \boldsymbol{\psi}_{\mathbf{2}}=\left[\begin{array}{l}
\psi_{2}^{(0)} \\
\psi_{2}^{(1)}
\end{array}\right]
$$

